\title{
CARDINAL INVARIANTS IN REMAINDERS AND VARIATIONS OF TIGHTNESS
}

\author{
A. V. ARHANGEL'SKII AND A. BELLA
}

(Communicated by Franklin D. Tall)

\begin{abstract}
In this paper certain invariants related to the tightness are considered. Particular attention is given to the set tightness. Relations between properties of remainders and compactifications of a metrizable space are also investigated.
\end{abstract}

In this paper we present some results concerning cardinal invariants of the type of tightness. In the first part we concentrate our attention on the set tightness. This invariant was originally introduced in [3] and it plays an important role in the class of pseudoradial spaces. Among other results, we show that for compact spaces tightness and set tightness coincide, and we give an example which clarifies that, in spite of the behaviour of the tightness, the set tightness can increase even under perfect maps. In the second part of the paper we prove that properties like countable tightness or sequentiality hold in a compactification of a metrizable space if and only if they hold in the remainder.

\section{DEFINITIONS AND FIRST RESULTS}

Throughout the paper all spaces are assumed to be at least Hausdorff. Our notation follows $[1,6] . X$ always denotes a topological space and $\tau$ an infinite cardinal number. $n w(X), w(X), \psi(X), \pi \chi(X), \chi(X)$, and $F(X)$ denote respectively the network weight, the weight, the pseudocharacter, the $\pi$-character, the character, and the supremum of the lengths of free sequences in $X$. The definition of free sequence is recalled below. If $\gamma$ is a family of subsets of $X$ then $\bar{\gamma}$ denotes the family $\{\bar{A}: A \in \gamma\}$.

The tightness of $X$, denoted by $t(X)$, is the smallest cardinal $\tau$ such that for any nonclosed set $A \subset X$ and any point $x \in \bar{A} \backslash A$ there exists a set $B \subset A$ satisfying $|B| \leq \tau$ and $x \in \bar{B}$.

The weak tightness (or c-tightness) of $X$, denoted by $w t(X)$, is the smallest cardinal $\tau$ such that for any closed set $F \subset X$ and any accumulation point $x \in F$ there exists a set $B \subset F \backslash\{x\}$ satisfying $|B| \leq \tau$ and $x \in \bar{B}$.

The set tightness of $X$, denoted by $t_{s}(X)$, is the smallest cardinal $\tau$ such that for any nonclosed set $A \subset X$ and any point $x \in \bar{A} \backslash A$ there exists a family

Received by the editors April 5, 1991.

1991 Mathematics Subject Classification. Primary 54A25, 54B10, 54D35, 54D40, 54D55.

Key words and phrases. Tightness, set tightness, cardinal invariants, remainder, compactification, topological groups, monolithic spaces. 
$\gamma$ of subsets of $A$ satisfying $|\gamma| \leq \tau$ and $x \in \bar{U} \backslash \backslash \bar{\gamma}$.

This invariant was originally called quasicharacter in [3], where it was introduced, and in fact the inequality $t_{s}(X) \leq \psi(X)$ always holds.

It is well known that replacing the expression "for any $x \in \bar{A} \backslash A$ " with "for some $x \in \bar{A} \backslash A$ " in the definition of tightness, we get the same cardinal invariant. A similar change for the set tightness leads to the definition of a new invariant which a priori is different from the previous one.

The partial set tightness of $X$, denoted by $e t_{s}(X)$, is the smallest cardinal $\tau$ such that for any nonclosed set $A \subset X$ there exists a family $\gamma$ of subsets of $A$ satisfying $|\gamma| \leq \tau$ and $(\overline{\bigcup \gamma} \backslash \bigcup \bar{\gamma}) \backslash A \neq \varnothing$.

Finally one more invariant follows by mimicking the definition of weak tightness.

The weak set tightness of $X$, denoted by $w t_{s}(X)$, is the sma!lest cardinal $\tau$ such that for any closed set $F \subset X$ and any accumulation point $x \in F$ there exists a family $\gamma$ of subsets of $F \backslash\{x\}$ satisfying $|\gamma| \leq \tau$ and $x \in \bar{U} \backslash \bigcup \bar{\gamma}$.

0.1. Proposition. For any space $X$ we have $w t_{s}(X) \leq e t_{s}(X) \leq t_{s}(X) \leq$ $w t(X) \leq t(X)$.

Proof. The only nontrivial inequality is the third. So let $A$ be a nonclosed subset of $A$ and $x \in \bar{A} \backslash A$. Put $F=\bar{A}$ and select a set $B \subset F \backslash\{x\}$ such that $x \in \bar{B}$ and $|B| \leq w t(X)$. For any $z \in B$ take an open set $U_{z}$ such that $z \in U_{z}$ and $x \notin \overline{U_{z}}$. The family $\gamma=\left\{U_{z} \cap A: z \in B\right\}$ satisfies $x \in \bar{U} \backslash \bigcup \bar{\gamma}$ and hence $t_{s}(X) \leq w t(X)$.

An example of a Lindelof regular space $X$ for which $w t(X)<t(X)$ is described in the next section.

If $X=\omega_{1} \times \omega \cup\{p\}$, topologized in such a way that any point in $\omega_{1} \times \omega$ is isolated and a local base at $p$ consists of the family $\left\{\{p\} \cup\left(\omega_{1} \backslash \alpha\right) \times(\omega \backslash n)\right.$ : $\left.(\alpha, n) \in \omega_{1} \times \omega\right\}$, we obtain a Lindelof regular space for which $t_{s}(X)<w t(X)$.

Surprisingly for the first two inequalities in the above proposition the situation is different, unless one wants to look for some rather pathological example.

0.2. Theorem. If $X$ is regular then $w t_{s}(X)=e t_{s}(X)=t_{s}(X)$.

Proof. Clearly it is enough to show that $t_{s}(X) \leq w t_{s}(X)$. Let $A$ be a non closed subset of $X$ and $x \in \bar{A} \backslash A$. Put $F=\bar{A}$, and select a family $\gamma$ of subsets of $F \backslash\{x\}$ in such a way that $|\gamma| \leq w t_{s}(X)$ and $x \in \overline{\cup \gamma} \backslash \bigcup \bar{\gamma}$. For any $B \in \gamma$ choose an open set $U_{B}$ such that $\bar{B} \subset U_{B}$ and $x \notin \overline{U_{B}}$. The family $\gamma^{\prime}=\left\{A \cap U_{B}: B \in \gamma\right\}$ satisfies the condition $x \in \overline{U \gamma^{\prime}} \backslash \bigcup \overline{\gamma^{\prime}}$ and therefore $t_{s}(X) \leq w t_{s}(X)$.

Before closing this section we mention a recent result of Juhàsz and Szentmiclossi which will be used later.

0.3. Proposition (see [8]). If $X$ is a compact space and there exists in $X$ a free sequence of length $\tau$, where $\tau$ is a regular cardinal and $\tau>\aleph_{0}$, then there exists in $X$ a converging free sequence of length $\tau$.

\section{AROUND SET TIGHTNESS}

The behaviour of the set tightness under the usual topological operations was studied in [5]. In particular, partial results were obtained in connection with 
the stability of set tightness in a product with a compact factor or under closed continuous maps.

In this section, among other results, a full solution of both of the above questions will be presented.

We begin with a nice consequence of Proposition 0.3, showing that in the class of compact spaces all the invariants in Proposition 0.1 coincide.

\subsection{Theorem. If $X$ is a compact space then $t(X)=t_{s}(X)$.}

Proof. According to Proposition 0.3 if in $X$ there is a free sequence of length $\tau^{+}$then there is also a free sequence of the same length which converges to a point. Assume by contradiction that $\tau=t_{s}(X)<t(X)$, and let $\xi$ be a free sequence of length $\tau^{+}$converging to a point $x$. If $A$ is a subset of $\xi$ such that $x \notin \bar{A}$ then it follows that $|A| \leq \tau$. Therefore, if $\gamma$ is a family of subsets of $\xi$ such that $|\gamma| \leq \tau$ and $x \notin \bigcup \bar{\gamma}$ then $x \notin \overline{U \gamma}$. This contradicts the definition of set tightness and the proof is complete.

Combining the above result with Corollary 2.7 in [5] we get

1.2. Corollary. If $X$ is compact then $t_{s}(X \times Y)=t_{s}(X) t_{s}(Y)$.

The above corollary can also be derived from the next more general statement.

1.3. Theorem. Let $X$ be regular and $f: X \rightarrow Y$ be a closed continuous map. If $t_{s}(Y) \leq \tau$ and each fibre of $f$ has set tightness not exceeding $\tau$ then $t_{s}(X) \leq \tau$.

Proof. Let $A$ be a nonclosed subset of $X$ and let $x \in \bar{A} \backslash A$. If $x \in$ $\overline{f^{-1}(f(x)) \cap A}$ then we find a family $\gamma$ in $f^{-1}(f(x)) \cap A$, and hence in $A$, satisfying $|\gamma| \leq \tau$ and $x \in \overline{\cup \gamma} \backslash \bigcup \bar{\gamma}$. Otherwise, we can assume that $f(x) \notin f(A)$ and consequently there exists a family $\gamma$ in $f(A)$ satisfying $|\gamma| \leq \tau$ and $f(x) \in \bar{U} \gamma \backslash \bigcup \bar{\gamma}$. Letting $\gamma^{\prime}=\left\{f^{-1}(B) \cap A: B \in \gamma\right\}$, we get a family in $A$ such that $(\overline{\bigcup \gamma} \backslash \bigcup \bar{\gamma}) \backslash A \neq \varnothing$ and therefore $e t_{s}(X) \leq \tau$. It is enough now to apply Proposition 0.2.

To show that the set tightness can increase even for perfect maps we need to recall an example constructed by Ranchin in [11].

Let $I$ be the unit segment and $A(I)=I_{1} \cup I_{2}$ be the Aleksandroff duplicate. Let $X$ be the space obtained from $A(I)$ by enlarging the topology in such a way that any countable subset of $I_{2}$ is closed in $X$. Then $X$ is a regular Lindelof space having $\psi(X)=t_{s}(X)=w t(X)=\aleph_{0}$ and $t(X)>\aleph_{0}$. Let $Y$ be the space obtained by collapsing $I_{1}$ to a point and observe that $Y$ is a perfect image of $X$. Since $Y$ is the one-point Lindelofication of a discrete set of cardinality $2^{\aleph_{0}}$, we clearly have $t_{s}(Y)>\aleph_{0}$.

Since any $G_{\delta}$ set is open in the space $Y$, the above construction also shows that a perfect image of a space of countable pseudocharacter can be a space without even the slightest trace of this property.

Since every target space can be represented as an image of a space of countable pseudocharacter under an open continuous mapping, we can conclude that an open continuous mapping can increase set tightness as much as we want.

The situation changes if we assume that the target space is countably compact.

Recall that a set $A$ in the space $X$ is said to be bounded provided that any continuous real-valued map on $X$ is bounded on $A$. 
1.4. Lemma (see [10 Theorem 2.1]). If $f: X \rightarrow Y$ is a closed continuous map and $Y$ is countably compact then each fibre of $f$ has the boundary bounded in $X$.

1.5. Lemma. If $f: X \rightarrow Y$ is a closed continuous map, $Y$ is countably compact, and any fibre of $f$ is bounded in $X$ then $X$ is pseudocompact.

Proof. Assume by contradiction that $X$ is not pseudocompact and select an unbounded continuous map $g: X \rightarrow \Re$. Choose a sequence $A=\left\{x_{n}: n \in \omega\right\}$ in $X$ so that $g\left(x_{n}\right)>n$ for any $n \in \omega$. Since any fibre of $f$ is bounded in $X$, it follows that the set $f(A)$ is infinite and thus has an accumulation point $y$. Because $g$ is bounded on $f^{-1}(y)$, there exists a neighbourhood $U$ of $f^{-1}(y)$ such that $g$ is also bounded on $U$. Observe now that, by the closedness of $f$, infinitely many points of $A$ must be in $U$, and this is a contradiction.

1.6. Theorem. If $f: X \rightarrow Y$ is a closed continuous map onto, where $X$ is Tychonoff, $\psi(X)=\aleph_{0}$, and $Y$ is countably compact then $Y$ is Frechet-Urysohn. Proof. Applying Lemma 1.4 we find a closed subset $Z$ of $X$ such that $f(Z)=$ $f(X)$ and each fibre of $\left.f\right|_{Z}$ is bounded in $Z$. From Lemma 1.5 we deduce that $Z$ is pseudocompact and, hence, being also of countable pseudocharacter and regular, it is first countable. The theorem now follows easily.

To conclude this section we state a result which shows that the behaviour of set tightness with respect to addition is better than the one of tightness (see [11]).

1.7. Theorem. Let $X$ be a space and $\mathscr{F}$ a closed cover of it. If $|\mathscr{F}| \leq \tau$ and $t_{s}(F) \leq \tau$ for any $F \in \mathscr{F}$ then $t_{s}(X) \leq \tau$.

Proof. Let $A$ be a nonclosed subset of $X$ and $x \in \bar{A} \backslash A$. If there exists an element $F \in \mathscr{F}$ such that $x \in \overline{A \cap F}$ then we are done. If $x \notin \overline{A \cap F}$ for any $F$ then it suffices to take $\gamma=\{A \cap F: F \in \mathscr{F}\}$.

An analogous result for tightness holds for example for $k$-spaces, but is not valid in general.

\section{TIghtNesS, SEQUENTIALITY, AND REMAINDERS}

In this section we investigate when a property of the type of tightness or sequentiality holds in a compactification provided that it holds in the remainder. In the sequel $Z$ always denotes a compactification of the space $X$.

2.1. Theorem. If $X$ is metrizable and $t_{s}(Z \backslash X) \leq \tau$ then $t(Z) \leq \tau$.

Proof. By virtue of Theorem 1.1 it is enough to show that $t_{s}(Z) \leq \tau$. To this end we need to verify that any subset $A$ of $Z$ is closed provided that for any family $\gamma$ of subsets of $A$ of cardinality not exceeding $\tau$ we have $(\bar{\bigcup} \backslash \bigcup \bar{\gamma}) \backslash A=\varnothing$. Thus let $x \in \bar{A}$. If $x \in X$ then, since $x$ has a countable base in $X$ and $X$ is dense in the regular space $Z, x$ has a countable base in $Z$ and consequently $x \in A$. If $x \notin A$ and $x \in \overline{A \backslash X}$ then the assumption that $t_{s}(Z \backslash X) \leq \tau$ implies $x \in A$. It remains to examine the case when $x \notin X$ and $A \subset X$. The closure in $Z$ of any countable subset of $A$ must be a subset of $A$, and consequently $A$ is countably compact. As $X$ is metrizable, it follows that $A$ is actually compact; therefore, $x \in \bar{A}$ cannot occur. The proof is complete.

The previous theorem leads to a slight generalization of Theorem 1.1. 
2.2. Corollary. If $X$ is the remainder of a compactification of a metrizable space then $t_{s}(X)=t(X)$.

2.3. Corollary. If $X$ is a metrizable space then $h \pi \chi(Z \backslash X)=t_{s}(Z \backslash X)$.

Proof. By Theorem 2.1 we have $t(Z)=t_{s}(Z \backslash X)$. Since $\overline{Z \backslash X}$ is compact, we have, by a theorem of Sapirovski (see [1]): $h \pi \chi(\overline{Z \backslash X})=t(\overline{Z \backslash X}) \leq t(Z)$. Thus we get $h \pi \chi(Z \backslash X) \leq t_{s}(Z \backslash X)$. The converse inequality is trivial.

A consequence of Corollary 2.3 is the following:

2.4. Corollary. If a topological group $G$ of countable tightness is the remainder in a compactification of a metrizable space then $G$ is metrizable.

The compactification in the above result, being the union of two metrizable spaces, is an Eberlein compact. In general it is not metrizable as the Aleksandroff duplicate of the circumference shows.

2.5. Lemma. If a compact space $X$ is the union of two dense metrizable subspaces then $X$ is metrizable.

Proof. Let $X=A \cup B$, where $A$ and $B$ are metrizable and $\bar{A}=\bar{B}=X$. Let $\mathscr{A}$ and $\mathscr{B}$ be $\sigma$-discrete bases of $A$ and $B$. Select two families $\widehat{\mathscr{A}}$ and $\widehat{\mathscr{B}}$ of open subsets of $X$ in such a way that the traces of them on $A$ and $B$ are $\mathscr{A}$ and $\mathscr{B}$. The families $\widehat{\mathscr{A}}$ and $\widehat{\mathscr{B}}$ are $\sigma$-disjoint, and therefore their union is a point countable base of $X$. The metrizability of $X$ now follows by a well-known result of Miscenko.

2.6. Corollary. If $X$ is a nowhere locally compact metrizable space and $Z \backslash X$ a topological group of countable tightness then $Z$ is metrizable.

Proof. By Corollary 2.4 the space $Z \backslash X$ is metrizable. Since $X$ is nowhere locally compact, it follows that $Z \backslash X$ is dense in $Z$. It is enough now to apply Lemma 2.5.

Observe that in the statement of Corollary 2.6 the assumption that $X$ is nowhere locally compact cannot be dropped. Look again at the Aleksandroff duplicate of a circumference.

We have, however, the following:

2.7. Corollary. If $X$ is separable metrizable and $Z \backslash X$ is a topological group of countable tightness and countable cellularity then $Z$ is separable metrizable.

Proof. In this case $Z \backslash X$ is separable metrizable. Then $Z$, being the union of two subspaces with a countable network, has a countable network. The compactness of $Z$ does the rest.

The next proposition generalizes a well-known formula for compact spaces to the remainder of a compactification of a metrizable space.

2.8. Theorem. If $X$ is metrizable then $t(Z \backslash X)=F(Z \backslash X)$.

Proof. By Theorem 5 in [4] we have $t_{s}(Z \backslash X) \leq F(Z \backslash X)$, and hence by Corollary $2.2 t(Z \backslash X) \leq F(Z \backslash X)$. On the other hand, it is clear that a regular uncountable free sequence of $Z \backslash X$ cannot have any complete accumulation point in $X$, while it must have a complete accumulation point in $Z$ (and hence in $Z \backslash X)$. This shows that $F(Z \backslash X) \leq t(Z \backslash X)$ and the proof is complete.

A result similar to Theorem 2.1 could hold for the property of sequentiality, but we do not have a full answer in ZFC. 
2.9. Theorem $\left(2^{\aleph_{0}}<2^{\aleph_{1}}\right)$. If $X$ is metrizable and $Z \backslash X$ is sequential then $Z$ is sequential.

Proof. It is enough to show that any sequentially closed subset of $Z$ is closed. Arguing as in Theorem 2.1, we can reduce the proof to checking that any relatively closed subset of $X$ which is sequentially closed in $Z$ is actually countably compact. Assume the contrary and let $N \subset A$ be a copy of the naturals relatively closed in $A$. Obviously $\bar{N} \neq N$ and therefore $\bar{N} \backslash N$ is a nonempty compact sequential space. Since $\bar{N}$ has weight not exceeding $2^{\aleph_{0}}$ and $\bar{N} \backslash N$ is sequential, it follows that $|\bar{N} \backslash N| \leq 2^{\aleph_{0}}$. By the Cech-Pospishil Theorem and $\left(2^{\aleph_{0}}<2^{\aleph_{1}}\right.$ ) there exists in $\bar{N} \backslash N$ a point of countable character in $\bar{N} \backslash N$ and hence in $\bar{N}$. Because $\bar{N} \cap A=N$, such a point belongs to $Z \backslash X$, and consequently there exists a sequence in $A$ converging outside $X$. This is in contrast with the fact that $A \subset X$, and the proof is complete.

The preceding theorem can also be proved assuming (MA), using a result of [9], or in ZFC assuming in addition that $|Z|<2^{\aleph_{1}}$.

It is natural to ask whether sequentiality can be replaced by some more general convergence property, for instance, pseudoradiality.

A space $X$ is said to be pseudoradial (or chain-net) provided that for any nonclosed subset $A$ of $X$ there exists a point $x \in \bar{A} \backslash A$ and a well-ordered (transfinite) sequence in $A$ converging to $x$. If the requirement of the previous definition is satisfied for every point $x \in \bar{A} \backslash A$ then $X$ is said to be radial.

2.10. Question. Is it true that if $X$ is metrizable and $Z \backslash X$ radial or pseudoradial then $Z$ is pseudoradial?

Recall that a space $X$ is said to be monolithic provided that $n w(\bar{A}) \leq|A|$ for any $A \subset X$.

Let us consider Isbell's space. Precisely, let $N$ be the set of natural numbers and fix an almost disjoint family $\mathscr{A}$ of subsets of $N$ such that $|\mathscr{A}|=2^{\aleph_{0}}$. Topologize the set $N \cup \mathscr{A}$ in such a way that any point of $N$ is isolated and a local base at the point $A \in \mathscr{A}$ consists of the sets of the form $\{A\} \cup(A \backslash F)$, where $F$ is a finite subset of $A$. The space so obtained is locally compact Hausdorff, and therefore its one-point compactification, say $Z$, is Hausdorff. The space $Z$ is also a compactification of $N$, which is metrizable, and $Z \backslash X$ is the one-point compactification of a discrete space of cardinality $2^{\aleph_{0}}$, which is monolithic. The space $Z$ instead is not monolithic.

By a result of Gruenhage, any compact monolithic space is pseudoradial (see a generalization in [2]) and the space in the previous example is sequential. These considerations lead us to formulate:

2.11. Question. Is it true that if $X$ is metrizable and $Z \backslash X$ is monolithic then $Z$ is pseudoradial?

2.12. Question. Is it true that if $X$ is a metrizable space and $Z \backslash X$ is a linearly ordered topological space then $Z$ is pseudoradial?

The previous example shows that the space $Z$, under the assumptions in Question 2.12, need not be radial.

Observe that Theorem 2.1, Corollary 2.2, and Theorem 2.9 are valid (with the same proofs) not only for metrizable spaces but for all bisequential Tychonoff spaces in which every countable compact subspace is compact. 
2.13. Proposition. If $X$ is a separable metrizable space then $n w(Z \backslash X)=w(Z)$; in particular, $n w(Z \backslash X)=w(Z \backslash X)$.

Proof. Since $X$ is separable, it follows that $n w(Z \backslash X)=n w(Z)$. The compactness of $Z$ does the rest.

2.14. Proposition. If $X$ is a separable metrizable space then $\psi(z, Z \backslash X)=$ $\chi(z, Z)$ for any point $z \in Z \backslash X$; in particular, $\psi(Z \backslash X)=\chi(Z \backslash X)$.

Proof. Let $\psi(z, Z \backslash X)=\tau$ and select a family $\left\{U_{\alpha}: \alpha \in \tau\right\}$ of open subsets of $Z \backslash X$ such that $\bigcap_{\alpha \in \tau} U_{\alpha}=\{z\}$. For any $\alpha$ take a set $V_{\alpha}$ open in $Z$ such that $U_{\alpha}=V_{\alpha} \cap(Z \backslash X)$. Since $X$ has countable base, $X$ can be covered by a countable family of closed subsets of $Z$ whose union misses $z$. If $\mathscr{W}$ denotes the set of the complements in $Z$ of the members of the previous family then $\mathscr{W} \cup\left\{V_{\alpha}: \alpha \in \tau\right\}$ is a local pseudobase at $z$ in $Z$. The compactness of $Z$ does the rest.

Observe that Proposition 2.14 is in general not true for a metrizable nonseparable space. To see this let $Z$ be the product of the unit interval $I$ and the one-point compactification $\omega_{1} \cup\{p\}$ of the discrete space $\omega_{1}$, and let $X=(I \backslash Q(I)) \times \omega_{1}$, where $Q(I)$ denotes the set of all rational numbers contained in $I$. If $z=(s, p)$, where $s \in I \backslash Q(I)$, then the family \{]$s-\frac{1}{n}, s+\frac{1}{n}[\times$ $\left.\left(\omega_{1} \cup\{p\}\right) \backslash X: n \in N^{+}\right\}$is a countable local pseudobase at $z$ in $Z \backslash X$ and hence $\psi(z, Z \backslash X)=\aleph_{0}$. On the other hand, since $Z \backslash X$ is dense in $Z$, we have $\chi(z, Z \backslash X)=\chi(z, Z)=\aleph_{1}$.

In connection with Proposition 2.13 we can formulate the following:

2.15. Question. Is it true that $n w(Z \backslash X)=w(Z \backslash X)$ holds for any metrizable space $X$ ?

To finish observe that Corollaries 2.2 and 2.3, Theorem 2.8, and Propositions 2.13 and 2.14 suggest that those relations between cardinal invariants, which are specific for the class of compact spaces, can be in general true even in the class of the remainders of compactifications of metrizable spaces.

\section{ACKNOWLEDGMENT}

The authors wish to thank the referee for the helpful comments.

\section{REFERENCES}

1. A. V. Arhangel'skii, Structure and classification of topological spaces and cardinal invariants, Russian Math. Surveys 33 (1978), 33-95.

2. A. V. Arhangel'skii, A. Bella, and F. Cammaroto, Weak monolithicity, pseudo radial and weakly radial spaces, Boll. Un. Mat. Ital. (7) 5 (1991), 339-344.

3. A. V. Arhangel'skii, R. Isler, and G. Tironi, On pseudo radial spaces, Comment. Mat. Univ. Carolin. 27 (1986), 137-154.

4. A. Bella, Free sequences in pseudo radial spaces, Comment. Mat. Univ. Carolin. 27 (1986), 163-170.

5. __ On set tightness and T-tightness, Comment. Mat. Univ. Carolin. 27 (1986), 805-814.

6. R. Engelking, General topology, PWN, Warsaw, 1977.

7. I. Juhàsz, Variations on tightness, Studia Sci. Math. Hungar. 24 (1989), 179-186.

8. I. Juhàsz and Z. Szentmiklossy, Converging free sequences in compact spaces, preprint. 
9. V. I. Malychin and B. E. Shapirovski, Martin's axiom and properties of topological spaces, Soviet Math. Dokl. 14 (1973), 1746-1751.

10. E. Michael, A note on closed maps and compact sets, Israel J. Math. 2 (1964), 173-176.

11. D. V. Ranchin, Tightness, sequentiality and closed coverings, Soviet Math. Dokl. 18 (1977), 196-200.

Department of Mathematics, Moscow State University, Moscow 117234, Republic of RUSSIA

Department of Mathematics, University of Messina, 98166 Messina, Italy

Current address: Department of Mathematics, University of Cagliari, via Ospedale 72, 09124 Cagliari, Italy

E-mail address: BELLA@vaxca2.unica.it 